\title{
Le benzodiazepine nel trattamento dell'ansia: profilo clinico e farmacoeconomico
}

Barbara Cascio*, Orietta Zaniolo§

\begin{abstract}
Anxiety disorders impose a significant economic burden on healthcare system, patients and society as a whole. A patient with anxiety disorders, especially with panic disorder, shows some difficulties to work or to maintain an acceptable social functioning, and, frequently, needs more medical care than a normal subject, often due to unnecessary or inappropriate diagnostic tests. Adequate psychiatric treatment of anxiety disorders can lead to higher direct specialists costs (psychiatric drugs and visits) but this may be offset by a decrease in non-specialist care costs and in indirect costs.

Benzodiazepines have been extensively used for the treatment of anxiety and related disorders since the 1960 s. Although new pharmacological and psychological treatments for anxiety are available, a lot of psychiatrists continue to endorse benzodiazepines as primary or adjunctive treatment for these disturbs, especially in the early or acute phases of the illness.

In Italy, benzodiazepines have been available also as generic drugs for some years. In order to be accepted for marketing, a generic drug has to demonstrate bioequivalence, considered a reliable proxy of therapeutic equivalence, with the standard formulation and its price has to be at least $20 \%$ cheaper than the originator drug. The use of generic drugs can contain drug expenditures and facilitate a competitive market. Despite that their prices are considerably higher than those of generics, branded benzodiazepines are still widely prescribed, because of prejudices and lack of information in the community of physicians and patients. In conclusion, benzodiazepines may treat anxiety disorders, mainly in early and acute phases, increasing quality of life and saving medical resources. The availability of generics allows for marginal reduction in initial investment in drug costs.
\end{abstract}

Keywords: anxiety disorders, panic, costs, benzodiazepines, generic drugs Farmeconomia e percorsi terapeutici 2005; 6 (4): 353-363

\section{INTRODUZIONE}

La stima della prevalenza delle patologie legate all'ansia risulta notevolmente complicata, soprattutto a causa della frequente comorbidità di disturbi d'ansia diversi (disturbo d'ansia generalizzato, attacchi di panico) e di disturbi depressivi [1]. Inoltre, le scale di misurazione utilizzate negli studi epidemiologici sono spesso poco affidabili [2]. Nel complesso, i disturbi d'ansia colpiscono circa il $25 \%$ delle persone nell' arco della loro vita $[3,4]$.

Uno studio statunitense che ha utilizzato $i$ criteri diagnostici definiti dal DSM-III (Diagnostic and Statistical Manual of Mental Disorders - III ed.), ha stimato che un soggetto su 20 sviluppa un disturbo d'ansia generalizzato nel corso della vita [5]. Un recente studio di coorte, che ha seguito soggetti affetti da disturbi depressivi e disturbi d'ansia per oltre
2 anni, ha riscontrato che la diagnosi di disturbo d'ansia generalizzato era confermata nel $49 \%$ di coloro per i quali era stata posta inizialmente [6].

Alcune recenti revisioni della letteratura hanno rilevato che il disturbo d'ansia generalizzato ha un'incidenza circa doppia nelle donne [7] e minore nei soggetti anziani [8].

Il disturbo di panico esordisce spesso intorno ai 20 anni (tra la tarda adolescenza e la parte centrale del quarto decennio di vita) [9]; la comparsa di attacchi di panico sporadici e di lieve entità in condizioni di stress particolari colpisce il 30-35\% della popolazione, mentre gli attacchi di panico di interesse clinico colpiscono il 2-3\% della popolazione con una particolare prevalenza tra le donne [4].

Uno studio australiano ha rilevato tassi di incidenza mensile per il disturbo di panico (con 
o senza agorafobia) dello $0,4 \%$ utilizzando i criteri diagnostici dell'ICD-10 (ICD-10 Classification of Mental and Behavioural Disorders - World Health Organization) e dello $0,5 \%$ servendosi invece dei criteri diagnostici del DSM-IV (Diagnostic and Statistical Manual of Mental Disorders - IV ed.) [10].

Da questi dati emerge che trattare correttamente i disturbi d'ansia costituisce uno sforzo non indifferente per la società soprattutto a causa dell'elevato costo di gestione, sia ospedaliero che territoriale, tipico delle patologie croniche caratterizzate da episodi acuti e improvvisi.

Nonostante, soprattutto fra le fasce più disagiate della popolazione, sia ancora elevata la percentuale di pazienti con disturbi psichiatrici che non ricorre a nessun tipo di trattamento, le diagnosi di questo tipo di patologie sono aumentate negli ultimi anni probabilmente grazie a una maggiore informazione e sensibilità nei confronti del problema.

In un momento di generale restrizione della spesa pubblica e di risorse ben definite, anche nel nostro Paese si comincia a valutare e a confrontare, da un lato, i costi delle patologie e, dall'altro, i costi di un intervento terapeutico adeguato.

In altri Paesi esistono già dati concreti su cui basarsi. Negli Stati Uniti, i costi associati ai disturbi d'ansia superano abbondantemente i 40 miliardi di dollari, secondo i dati relativi al 1990, e i 60 miliardi di dollari con riferimento all'anno 1998, circa un terzo della spesa complessiva per la salute mentale [11]. In Italia mancano ancora dati precisi e il trasferimento di queste valutazioni alla situazione italiana non è facilmente attuabile, data la diversa struttura del sistema assistenziale sanitario dei due paesi. Da alcuni dati pubblicati dall'Associazione per la Ricerca sulla Depressione [12], nel nostro Paese, il costo sociale di depressione, ansia e attacchi di panico corrispondeva nel 1994 a circa 8.000 miliardi di lire l'anno.

La gestione degli attacchi di panico e del disturbo d'ansia generalizzato comporta l'adozione di strategie terapeutiche personalizzate, per lo più complesse, di lunga durata $\mathrm{e}$ costose.

Ogni paziente affetto da disturbi d'ansia si trova ad effettuare un proprio percorso diagnostico-terapeutico, la cui appropriatezza è una condizione fondamentale per garantire la massima efficienza dell' assistenza sanitaria. Per ottimizzare questo percorso è necessario produrre il massimo beneficio possibile, tenendo in giusta evidenza i bisogni sanitari oggettivi e soggettivi delle singole persone e le risorse disponibili. Il beneficio sanitario si può tradurre in un corrispondente beneficio economico grazie al parziale risparmio in termini di consumo di risorse indotto dalla presenza della patologia stessa.
Le linee guida implementate dall' American Psychiatric Association (APA) raccomandano l'utilizzo delle benzodiazepine nel trattamento dei disturbi d'ansia e da attacchi di panico in fase acuta, grazie al rapido e potente sollievo dei sintomi che questa classe di farmaci è in grado di indurre. Per la terapia di mantenimento, invece, viene consigliato l'utilizzo degli inibitori selettivi del reuptake di serotonina o di altri agenti antidepressivi, a causa dei fenomeni di dipendenza e di tolleranza a cui è spesso associata l'assunzione delle benzodiazepine nel lungo periodo [13].

Scopo di questo lavoro è quello di analizzare la formazione dei costi legati ai disturbi d'ansia e alla loro gestione.

Questo permette di valutare, in base alla letteratura disponibile, in quale misura la terapia farmacologica, in particolare il trattamento a base di benzodiazepine, alleviando i sintomi legati alla patologia e riducendo la frequenza di comparsa di attacchi acuti, possa influenzare tale spesa.

\section{PROFILO CLINICO DELLE BENZODIAZEPINE}

Da secoli l'uomo ricerca medicinali che riducano l'ansia e lo sollevino dall' angoscia che gli eventi di vita possono provocare; fin dall'antichità inoltre sono state ricercate sostanze che inducessero e mantenessero il sonno.

Inizialmente furono impiegati farmaci che utilizzavano l'effetto sedativo per mascherare l'ansia. Dagli anni '60 l'introduzione sul mercato delle benzodiazepine (BDZ) ha rivoluzionato il trattamento dell'ansia; si osservò infatti per la prima volta una reale azione ansiolitica, la riduzione dei sintomi non era cioè imputabile ad uno stato di sedazione indotta.

Le BDZ si sono subito rivelate sostanze migliori per efficacia, tollerabilità e sicurezza. Questa maneggevolezza, associata alla loro azione terapeutica su un esteso gruppo di disturbi, fece sì che il loro uso si diffondesse molto rapidamente e portò le $\mathrm{BDZ}$ ad essere tra i farmaci più prescritti al mondo.

\section{Meccanismo d'azione}

Le BDZ potenziano la funzione delle sinapsi GABAergiche attraverso una facilitazione dell'azione inibitoria del GABA (acido $\gamma$-aminobutirrico), il principale neurotrasmettitore inibitorio del sistema nervoso centrale dei mammiferi [14]. Il sito specifico di legame del GABA è situato all'interno di un complesso macromolecolare, il complesso recettoriale GABA A. A tale complesso si legano varie sostanze e farmaci di interesse in psichiatria, agonisti (BDZ), agonisti parziali, agonisti inversi e antagonisti. Questi agenti, con la loro diversa azione sulla trasmissione GABAergica centrale, sono importanti mediatori di attività del 
sistema nervoso centrale molto diverse tra loro [15]. Le principali differenze tra benzodiazepine, che ne determinano anche il diverso profilo terapeutico e di sicurezza, riguardano essenzialmente l'emivita e i processi metabolici di eliminazione dal plasma; molte benzodiazepine sono trasformate dai sistemi enzimatici mitocondriali del citocromo P450, con formazione di uno o più metaboliti, talvolta attivi, mentre altre sono inattivate principalmente per coniugazione.

\section{Efficacia clinica: indicazioni $e$ posologie}

L'impiego delle BDZ è diffuso in psichiatria come in altri vari settori specialistici, ma in particolare in medicina generale. Il medico di base infatti è spesso tra i primi prescrittori; frequentemente è poi il paziente che, trovandosi bene con il farmaco, continua a richiedere la sua prescrizione.

Le BDZ hanno un alto "indice di gradimento" terapeutico per varie ragioni. Innanzitutto per la loro rapida azione terapeutica, riducendo velocemente i sintomi hanno un forte rinforzo positivo per successive somministrazioni. Da qui però nasce spesso anche il loro cattivo uso o abuso. Una seconda ragione è data dalla facilità dell'utilizzo e dalla loro maneggevolezza. La terza ragione è la ridotta presenza di effetti collaterali. Infine, agevola il loro uso l'ampio margine terapeutico rispetto a rischi di tossicità e sovradosaggio.

Le BDZ agiscono direttamente e rapidamente sul sintomo ansia, pertanto sono indicate nella prima fase di trattamento in attesa che si esplichi l'efficacia della terapia patogenetica, mediata dalla neurotrasmissione serotoninergica e noradrenergica.

Nel trattamento acuto o subacuto con queste molecole si determinano importanti variazioni farmacodinamiche, quali un rapido e deciso innalzamento della soglia eccitatoria, che permettono di prevenire o controllare gli episodi di ansia acuta e di stress senza l'induzione di fenomeni di tolleranza o di dipendenza. Ciò risulta molto utile anche nelle fasi iniziali di un trattamento con antidepressivi, per ottenere un primo effetto terapeutico nel periodo di latenza relativamente lungo che caratterizza l'azione di queste molecole. Oggi la prescrizione medica di benzodiazepine è motivata da [16]: - ansia transitoria, reattiva o situazionale senza che si possa identificare un vero e proprio disturbo d'ansia fino a quadri di agitazione psicomotoria;

- ansia associata o secondaria ad una condizione medica generale dove l'obiettivo non è quello di eliminare il fisiologico vissuto d'ansia e preoccupazione connesso alla patologia fisica, ma solo di controllare l'emergenza di una sintomatologia ansiosa di entità tale da aggravare il decorso spontaneo della malattia di base;

- ansia associata ad altri disturbi psichiatrici (disturbi somatoformi, ipocondria, disturbi dell'umore, disturbi psicotici);

- disturbi d'ansia (disturbo d'ansia generalizzata, disturbo da attacchi di panico con o senza agorafobia, fobia semplice, fobia sociale, disturbo ossessivo-compulsivo, disturbo da stress acuto e post traumatico da stress)

- disturbi del sonno primitivi o secondari.

Rispetto alla sintomatologia ansiosa la scelta del trattamento farmacologico non differisce nei disturbi d' ansia primitivi rispetto a quelli associati.

È una convinzione diffusa quella che esista una sostanziale sovrapponibilità tra le varie BDZ, convinzione che si fonda su una visione incompleta e limitata delle loro caratteristiche.

L'eterogeneità della loro azione a livello clinico deriva da un effetto differenziato delle molecole a livello cerebrale, in relazione alle diversità intrinseche alle sottopopolazioni di recettori GABAA.

Tutte le BDZ possiedono intrinsecamente effetti ansiolitici (quando si realizza solo una riduzione dell'emozione ansiosa), sedativi (quando alla prima azione si associa una compromissione del livello di vigilanza e di efficienza psicomotoria) e ipnotici (quando la sedazione è di entità tale da indurre l'addormentamento). Si tratta sempre di azioni dose-dipendenti, e ciò che distingue le BDZ in ansiolitiche e ipnotiche è la variabile affinità per sottopopolazioni di recettori, che determina la differenza di dosaggio necessaria a passare dall'effetto ansiolitico a quello ipnotico [17].

Schematicamente, si può fare riferimento a una classificazione delle BDZ in base ai loro effetti clinici principali (Tabella I) .

Nella scelta del trattamento ansiolitico la conoscenza delle caratteristiche cinetiche di una BDZ è uno dei parametri fondamentali [18];

\begin{tabular}{cccc}
$\begin{array}{c}\text { Effetto } \\
\text { ansiolitico }\end{array}$ & $\begin{array}{c}\text { Effetto } \\
\text { ipnotico }\end{array}$ & $\begin{array}{c}\text { Effetto } \\
\text { anticonvulsivante }\end{array}$ & $\begin{array}{c}\text { Effetto } \\
\text { miorilassante }\end{array}$ \\
\hline $\begin{array}{ccc}\text { Clordiazepossido } \\
\text { Diazepam }\end{array}$ & $\begin{array}{c}\text { Flurazepam } \\
\text { Lormetazepam }\end{array}$ & $\begin{array}{c}\text { Clonazepam } \\
\text { Diazepam } \\
\text { Delorazepam }\end{array}$ & $\begin{array}{c}\text { Nitrazepam } \\
\text { Lobazam }\end{array}$ \\
Alprazolam & Flunitrazepam & & \\
Bromazepam & Triazolam & & \\
Clorazepato & Estazolam & & \\
Oxazepam & & & \\
Prazepam & & \\
Etizolam & & \\
Clotiazepam & & \\
\hline
\end{tabular}

Tabella I

Classificazione delle benzodiazepine in base ai loro effetti clinici principali 


\begin{tabular}{cccc}
\hline $\begin{array}{c}\text { Emivita } \\
\text { lunga }\end{array}$ & $\begin{array}{c}\text { Emivita } \\
\text { intermedia }\end{array}$ & $\begin{array}{c}\text { Emivita } \\
\text { breve }\end{array}$ & $\begin{array}{c}\text { Emivita } \\
\text { ultrabreve }\end{array}$ \\
\hline Diazepam & Lorazepam & $\begin{array}{c}\text { Etizolam } \\
\text { Delorazepam }\end{array}$ & $\begin{array}{c}\text { Bromazepam } \\
\text { Oxazolam }\end{array}$ \\
$\begin{array}{c}\text { Clurazepam } \\
\text { Prazepam }\end{array}$ & $\begin{array}{c}\text { Exazepam } \\
\text { Estazolam }\end{array}$ & $\begin{array}{c}\text { Alprazolam } \\
\text { Lormetazepam }\end{array}$ \\
Flunitrazepam & & & \\
Clobazam & & & \\
Nitrazepam & & & \\
Clonazepam & & & \\
\hline
\end{tabular}

\section{Tabella II}

Classificazione delle benzodiazepine in base alla durata della loro emivita

tra queste primaria importanza riveste l'emivita plasmatica, ossia il tempo di dimezzamento della concentrazione ematica. Si tratta di un parametro che ha un ruolo rilevante nella gestione di un trattamento con BDZ, in quanto in rapporto diretto con la durata dell' effetto terapeutico e degli effetti collaterali.

\section{Tollerabilità e sicurezza}

Una delle caratteristiche principali delle BDZ, alla base della loro ampia diffusione, è l'ottima tollerabilità. Sul piano somatico generale non sono noti specifici effetti tossici a livello epatico, renale, cardiaco, emopoietico e coagulativo. La stessa tossicità cerebrale acuta da sovradosaggio, pur nella sua reale pericolosità, si associa spesso ad un ampio e variabile grado di tolleranza individuale. Tuttavia esistono degli effetti delle BDZ che, anche se apparentemente non drammatici, influiscono negativamente sulla qualità di vita dei soggetti che le assumono per periodi prolungati. Gli effetti collaterali delle BDZ sono in genere dose-dipendenti. Comunemente si riducono di intensità o addirittura scompaiono proseguendo la terapia o riducendo la dose di farmaco somministrato.

In generale sono controindicate nel caso di preesistente sofferenza somatica per lo più di natura cerebrale o neuromuscolare, per un effetto peggiorativo su una funzionalità già di per sé deficitaria.

Gli effetti collaterali si dividono in effetti sulla sfera cognitiva, sulla performance psicomotoria e di tipo neuromuscolare. Tra i primi i più comuni sono la sonnolenza, le vertigini, la riduzione delle capacità di concentrazione e di memorizzazione, sia come difficoltà di fissazione che di rievocazione. Tale effetto è più marcato nel soggetto anziano a causa sia di un rallentato metabolismo epatico che di una fisiologica riduzione della trasmissione acetilcolinica. La tendenza all' accumulo ne amplifica gli effetti negativi sulla sfera cognitiva per cui si richiede cautela nell'uso.

Sul piano della performance psicomotoria le BDZ esercitano un effetto inibitorio che si esprime attraverso una riduzione del livello di vigilanza e un ritardo nell'esecuzione di compiti mentali o motori di diversa complessità.

L'inibizione delle BDZ sui centri motori cerebrali determina un differente grado di riduzione del tono muscolare. Le ripercussioni funzionali più comuni sono rappresentate dall'astenia e dall'affaticabilità durante sforzi fisici o compiti motori. Una controindicazione assoluta all'uso delle BDZ è la presenza di patologie deficitarie primitive del tono muscolare, come ad esempio la miastenia.

Molto rare sono le reazioni paradosse con irritabilità e aggressività sino alla agitazione psicomotoria.

Devono essere anche considerati fenomeni non infrequenti quali la riduzione di vitalità, l'impoverimento relazionale e l'influenza negativa sul tono dell'umore.

Nonostante la loro maneggevolezza e la loro alta tollerabilità, esistono alcune condizioni cliniche in cui le BDZ devono essere impiegate con cautela. In particolare nei soggetti anziani si deve tenere in considerazione la possibile riduzione del metabolismo epatico, con rischio di accumulo in caso di somministrazioni ripetute. In questi soggetti, più ancora che in altri, non si deve nemmeno trascurare l'effetto negativo sulle funzioni cognitive [19].

Se le BDZ vengono interrotte bruscamente, possono comparire sintomi da sospensione. Nella sindrome rebound si assiste alla ripresa, sospendendo una BDZ, dei sintomi dell'ansia con maggior intensità di prima del trattamento [20].

Quando le BDZ vengono assunte a dosi superiori al normale o per lunghi periodi di tempo si può instaurare una dipendenza fisica. Alla sospensione si presenta una sintomatologia non presente precedentemente all'inizio della terapia. I segni e i sintomi di astinenza possono comprendere tachicardia, ipertensione, crampi muscolari, ansia, insonnia, panico, atassia, agitazione psicomotoria, disturbi confusionali fino a crisi convulsive. Le reazioni di astinenza hanno un picco più rapido e intenso per le BDZ a breve emivita [21].

Nei pazienti in cui il quadro ansioso si presenta in comorbidità con patologie organiche, assume particolare rilevanza la sicurezza d'uso, comprendente anche il potenziale relativamente basso di interazioni farmacologiche, fattore importante nei pazienti internistici, spesso in regime politerapico.

\section{ANALISIFARMACOECONOMICA}

\section{Formazione dei costi di management dei disturbi d'ansia}

La gestione del paziente con disturbi d'ansia comporta costi legati direttamente alla cura della patologia e costi indiretti legati 
principalmente alla perdita di produttività lavorativa del paziente e, in molti casi, degli altri componenti del nucleo familiare.

Greenberg e colleghi hanno stimato che, negli Stati Uniti, circa l' $85 \%$ dei costi totali legati ai disturbi d'ansia (circa 42,3 miliardi di dollari l'anno) sono imputabili a costi diretti (visite psichiatriche, non psichiatriche, ricoveri e trattamento farmacologico) e il $13 \%$ a costi indiretti (perdita di lavoro e aumento di rischio di mortalità) [11].

I costi diretti psichiatrici rappresentano circa il $31 \%$ dei totali (di cui circa la metà dovuti alle spese di ricovero), mentre circa il 54\% della spesa totale è costituita dall'assistenza non psichiatrica: i pazienti ansiosi e depressi non utilizzano, infatti, solo i servizi specialistici, ma sono tra i maggiori utilizzatori di visite dal medico di medicina generale, di esami di laboratorio e di visite specialistiche di natura non psichiatrica. Essi hanno in media anche degenze ospedaliere più lunghe.

Ciò significa che a parità di patologia organica, un paziente depresso o affetto da disturbi d'ansia consuma più risorse sanitarie. I costi indiretti legati ai disturbi d'ansia sono dovuti alla perdita di giorni di lavoro, unita alla perdita di qualità delle prestazioni lavorative (circa 10\% del costo totale), e in misura minore alla premorienza per suicidio (circa 3\% del totale) [11].

La stima del costo di malattia, soprattutto per le patologie psichiatriche, è sempre complessa e conduce frequentemente a risultati controversi. Ad esempio, un altro studio svolto negli Stati Uniti, ha rilevato, a fronte di risultati simili in termini di costo globale (46,6 vs. 42,3 miliardi di dollari), una diversa ripartizione fra costi diretti e indiretti, stimando questi ultimi addirittura pari al $77 \%$ dei totali [22]. Il differente risultato è, verosimilmente, imputabile sia a una sottostima dei costi diretti che a una difficoltà intrinseca nel calcolare con precisione i costi indiretti.

$\mathrm{Al}$ di là del contrasto fra i risultati ottenuti dalle diverse stime, l'impatto a livello occupazionale è notevole, infatti i disturbi psichiatrici sono considerati fra le patologie più frequentemente responsabili della perdita di giorni lavorativi e della riduzione di produttività.

Anche nelle fasi asintomatiche o con sintomatologia lieve, il rendimento professionale è minore e il lavoro risulta dequalificato, limitando l'individuo nelle sue possibilità di carriera e di soddisfazione personale.

Inoltre, dai dati raccolti da alcune associazioni di pazienti è emerso che per ogni paziente, sono coinvolti almeno due/tre familiari o amici, i quali, in molti casi, sono costretti a impegnare ore lavorative e tempo libero per seguire e sostenere il paziente.

Altri importanti costi indiretti sono quelli legati alla mortalità anticipata per suicidio.

\section{Impatto dei disturbi d'ansia sulla qualità di vita}

I disturbi d'ansia, e le patologie psichiatriche in generale, incidono pesantemente sulla qualità di vita del paziente e dei suoi familiari.

Questo costituisce un costo intangibile, la cui misurazione è assai complessa a causa della soggettività e della variabilità con cui una condizione patologica può influenzare la vita personale e familiare del paziente e delle difficoltà oggettive nel monetizzare questo impatto; per tali ragioni nelle stime di costo di malattia questa tipologia di costo viene purtroppo trascurata.

Una revisione di studi epidemiologici e clinici ha indagato la riduzione della qualità di vita e del comportamento psico-sociale dei soggetti affetti da diversi disturbi d'ansia.

Fra i soggetti affetti da disturbo da attacchi di panico, il $35 \%$ prova uno scarso livello di benessere fisico e il $38 \%$ ha una percezione molto negativa della propria sfera emozionale e delle proprie funzioni sociali. I risultati dei pazienti che non soffrono di questa patologia sono notevolmente migliori sia per quanto concerne l'ambito fisico che l'ambito psicosociale ( $24 \%$ e $16 \%$ rispettivamente).

Anche i pazienti con attacchi di panico con frequenza troppo bassa per porre diagnosi di disturbo da attacchi di panico, secondo i criteri del DSM-IV, hanno riportato una percezione negativa del loro ruolo occupazionale, psicosociale e fisico; tale influenza è risultata di entità intermedia rispetto ai pazienti con disturbo da panico e ai pazienti senza alcun disturbo psichiatrico [23].

Lo strumento più utilizzato per misurare la qualità di vita è il questionario generale Medical Outcomes Survey Short-form 36 (SF-36). Esso è diviso in otto domini che indagano quattro aspetti della funzionalità fisica e quattro della sfera emozionale.

Numerosi studi [24-28] hanno confrontato il livello di qualità di vita di pazienti affetti da disturbo da attacchi di panico con quello di soggetti senza alcun disturbo particolare e con quello di pazienti affetti da depressione o da condizioni patologiche croniche (ipertensione, diabete, artrite).

Dai risultati di questi trial è emerso che $\mathrm{i}$ punteggi dello SF-36 dei pazienti con attacchi di panico sono in media decisamente inferiori a quelli dei soggetti senza condizioni patologiche particolari, sia per quanto riguarda la sfera fisica che psico-sociale; la qualità di vita di questi pazienti, anche se marcatamente più bassa della media, risulta però relativamente migliore di quella dei pazienti affetti da depressione o da condizioni patologiche croniche, soprattutto dal punto di vista físico.

Dalla stessa revisione della letteratura è emerso che anche gli altri disturbi d'ansia, come la fobia sociale, il disturbo da stress post- 
traumatico e il disturbo d'ansia generalizzato, interferiscono notevolmente con la vita relazionale e professionale del paziente $\mathrm{e}$, in molti casi, dei suoi familiari.

Negli ultimi anni, un crescente numero di trial clinici ha inserito la misura della qualità di vita fra gli outcome principali per stabilire l'efficacia di una terapia; questo è particolarmente riscontrabile fra gli studi condotti sul trattamento del disturbo da attacchi di panico.

Questa scelta comporta l'utilizzo di uno strumento di valutazione di questo parametro che racchiuda in sé alcune caratteristiche indispensabili per l'ottenimento di dati completi e realistici: obbiettività, sensibilità, affidabilità e interpretabilità.

Gli strumenti che maggiormente rispondono a queste caratteristiche sono, oltre allo SF36, la Sheehan Disability Scale [29], la Social Adjustment Scale e il Quality of Life Enjoyment and Satisfaction Questionnaire [30].

Uno dei domini più importanti considerati dalle sopracitate scale di valutazione è quello relativo all'ambito professionale e alle attività quotidiane; ne consegue che un miglioramento significativo degli score di questi strumenti di valutazione si traduca in un miglioramento anche delle prestazioni lavorative, della concentrazione, della soddisfazione personale. Ciò implica, oltre ad un guadagno in termini di benessere psico-sociale per il paziente, anche una netta riduzione dei costi indiretti collegati alla patologia e gravanti sul paziente, sul suo nucleo familiare e sull'intera collettività.

\section{Conseguenze economiche dell'uso dei farmaci ansiolitici}

Da uno studio epidemiologico pubblicato nel 1994 e svolto a cura dell'Organizzazione Mondiale della Sanità su un campione di circa 80 mila soggetti, fra pazienti e medici di base, è emerso che fra le persone affette da depressione o da sintomatologia associate ad ansia o attacchi di panico, solo una minoranza (circa il 18\%) consulta lo specialista di competenza [31]. Probabilmente la causa di questa situazione è da ricercare in una disinformazione di base unita a un pregiudizio che ancora circonda le malattie psichiatriche; questo porta inevitabilmente al fatto che solo un'esigua percentuale di pazienti viene curata in maniera adeguata, con il conseguente aumento dei costi associati alla patologia non controllata e dei costi relativi all'assistenza non psichiatrica (visite dal medico di medicina generale, acquisto di farmaci non specifici, ecc.).

In Ungheria è stato svolto uno studio proprio al fine di indagare in che misura l'utilizzo di una terapia psichiatrica specifica influisca sul consumo delle risorse sanitarie generiche, come il numero di visite mediche non specialistiche o l'utilizzo di farmaci non psichiatrici, da parte di pazienti affetti da disturbi d'ansia o da disturbi dell'affettività [32].

Il trial ha indagato anche l'impatto della terapia psichiatrica sui costi indiretti associati a tali patologie. Allo studio hanno partecipato 12 medici di medicina generale, i quali hanno reclutato 2000 pazienti in modo random e indipendentemente dal sospetto che essi avessero patologie psichiatriche; di questi, 1815 hanno accettato di partecipare al trial. Dei primi mille pazienti arruolati, 151 rispondevano positivamente ai criteri stabiliti dal DSM-III per i disturbi d'ansia e del comportamento (utilizzo del questionario Diagnostic Interview Schedule - DIS).

Di questi, sei avevano solo agorafobia lieve e dieci erano già in cura presso uno psichiatra; ai restanti 135 venne offerta assistenza psichiatrica (trattamento per un anno in una sede territoriale da parte di alcuni psichiatri altamente qualificati), che fu accettata da 51 pazienti; 55 pazienti si rivolsero a servizi psichiatrici esterni al trial e i rimanenti rifiutarono il trattamento. I pazienti arruolati successivamente ai primi mille seguirono lo stesso iter procedurale, ma i soggetti rispondenti ai criteri per le patologie in analisi ( $\mathrm{N}$ : 75) vennero considerati pazienti-controllo e mantenuti con il trattamento abitualmente prescritto dal medico di medicina generale.

Al momento dell' arruolamento, dopo un anno e dopo due anni di osservazione, ai pazienti è stato chiesto di completare il questionario Quality of Life in Depression Scale.

I costi considerati nello studio sono quelli diretti sanitari e quelli indiretti. Il calcolo dei primi è stato effettuato in base al numero di visite mediche (non psichiatriche) svolte per paziente, al numero di giorni di ospedalizzazione, al costo farmaceutico (non psichiatrico) e degli esami di laboratorio. I costi indiretti sono stati stimati sulla base del numero di giorni di lavoro persi per malattia.

Dall' analisi dei dati raccolti è emerso che il numero di visite mediche per i pazienti trattati, superiore durante il primo anno di osservazione, nel corso del secondo anno di analisi decresce in misura maggiore rispetto agli altri gruppi. Confrontando i dati relativi al primo e al secondo anno per i pazienti trattati, gli autori hanno osservato anche una riduzione significativa nel numero medio di giorni di ospedalizzazione. I costi farmaceutici non psichiatrici risultano ridotti in modo statisticamente significativo nei gruppi trattati e non statisticamente significativo nel gruppo che rifiuta il trattamento; nel gruppo controllo tali costi sono aumentati.

Nei gruppi trattati si è osservata una diminuzione del $37 \%$ della spesa relativa ai farmaci non psichiatrici (primo anno vs secondo anno di trattamento); tale risparmio può in parte con- 
trobilanciare la spesa per i farmaci psichiatrici che, per i pazienti trattati rappresenta un costo da sommare al costo totale di prescrizione; lo stesso discorso vale per il costo relativo alle visite psichiatriche che il paziente in trattamento, a differenza dei soggetti non trattati, sostiene: la riduzione del numero di visite non specialistiche o di giorni di lavoro persi dovrebbe ampiamente bilanciare questa voce di costo. Nel gruppo controllo, infatti, si assiste a un aumento del $182 \%$ nel numero di giorni persi per malattia, a fronte di una diminuzione del $56 \%$ e del $62 \%$, rispettivamente, per i pazienti trattati internamente e esternamente.

Questo studio, seppure con grossi limiti dovuti all'assegnazione non random dei pazienti alle diverse opzioni terapeutiche e alle diversità fra le caratteristiche basali dei gruppi, sottolinea come un accurato trattamento psichiatrico può tradursi in un miglioramento della salute del paziente e della sua qualità di vita, senza un significativo aumento delle risorse sanitarie, in quanto i costi per il trattamento specialistico sono in parte controbilanciati dalla riduzione dei costi indiretti o dei costi diretti non psichiatrici.

È necessario comunque valutare attentamente il quadro clinico e sociale del paziente; l'uso improprio di un farmaco, per le conseguenze che ne derivano, rappresenta infatti un importante problema socio-sanitario, tanto sul piano clinico, quanto sul piano psico-sociale, interessando tutti gli strati sociali e ogni fascia d'età, soprattutto quella evolutiva.

Appare molto difficile calcolare in modo preciso il costo legato al danno provocato dall'uso sbagliato di un farmaco, soprattutto per i diversi livelli di rischio che questo può comportare, l'impatto più o meno rilevante che può avere sulla qualità di vita e sul rendimento lavorativo, e le diverse conseguenze che può comportare sul piano emotivo.

In ogni caso il costo socio-economico legato all'abuso di un farmaco, soprattutto psichiatrico, è spesso molto elevato [33].

\section{Il costo della terapia con benzodiazepine}

In Italia i derivati benzodiazepinici non sono prescrivibili in regime di rimborsabilità, e quindi da sempre rappresentano una spesa a carico del paziente.

La spesa sanitaria privata, cioè quella a carico del paziente, può però essere notevolmente ridotta grazie alla possibilità di scegliere il farmaco generico (o farmaco equivalente).

Un farmaco generico deve essere un equivalente farmaceutico del prodotto originatore, cioè deve avere la stessa composizione qualiquantitativa in principio attivo, la stessa forma farmaceutica, lo stesso dosaggio e la stessa via di somministrazione. Esso per definizione deve avere anche lo stesso profilo di efficacia e di tollerabilità dell'originatore, quindi la stessa biodisponibilità sistemica; deve cioè essere un equivalente terapeutico oltre che farmaceutico.

Poiché le caratteristiche chimico-fisiche degli eccipienti e delle tecnologie farmaceutiche utilizzate per preparare una specifica forma farmaceutica possono condizionare la velocità di passaggio in soluzione e, nel caso di forme solide, la velocità di disgregazione, per commercializzare un medicinale equivalente è necessario effettuare studi di biodisponibilità in vivo e testarne direttamente la bioequivalenza con il farmaco di riferimento. Gli studi di bioequivalenza sono basati sul confronto statistico di parametri farmacocinetici che caratterizzano la biodisponibilità dei due prodotti: il parametro AUC (area sotto la curva concentrazione-tempo del principio attivo) come indicatore della quantità di farmaco biodisponibile, Cmax (concentrazione di picco massimo) e Tmax (tempo di picco massimo) come indicatori della velocità con cui esso è disponibile. Due farmaci sono definiti bioequivalenti se si può determinare, con un buon livello di confidenza (fissato generalmente al 90\%), che la differenza tra le loro biodisponibilità oscilla entro un range predefinito in modo convenzionale come "intervallo accettabile" di bioequivalenza. Tale intervallo è così fissato dall'EMEA: la AUC media del prodotto testato può essere da 0,8 a 1,25 volte quella del prodotto di riferimento (la differenza non può comunque superare il $20 \%$ se si considerano i parametri normalizzati per il parametro della formulazione di riferimento).

Lo studio della bioequivalenza è importante sia come misura in vivo della qualità di una nuova forma farmaceutica ad uso sistemico, sia come stima surrogata della sua equivalenza terapeutica con la formulazione di riferimento, al fine di fornire al medico e ad altri decisori garanzie di prescrivibilità e di interscambiabilità [34].

Il mercato dei generici in Italia si è sviluppato a partire da alcune innovazioni normative precedenti al decreto del 20 maggio 2005, introdotte al fine di razionalizzare e contenere la spesa farmaceutica a carico del SSN.

Gli obiettivi principali della politica del generico sono di favorire un mercato farmaceutico maggiormente aperto alla concorrenza, di innescare una dinamica di riduzione dei prezzi dei medicinali e di consentire significativi risparmi sulla spesa farmaceutica.

Per conseguire tali obiettivi la legge prevede che il prezzo del generico sia almeno inferiore del $20 \%$ a quello del prodotto "originatore", il quale può, a sua volta, essere ridotto di prezzo, anche sotto il livello dei generici, innescando in tal modo una dinamica di 
prezzi al ribasso grazie alla possibilità di entrare in un mercato concorrenziale [35].

Nel tentativo di limitare la spesa sanitaria privata il Decreto Legge n. 87, emanato il 27 maggio 2005 dal Governo italiano su proposta del ministro della Salute Francesco Storace, prevede l'invariabilità dei prezzi dei farmaci in fascia $\mathrm{C}$ fino a gennaio $2007 \mathrm{e}$ la possibilità per i farmacisti di applicare uno sconto non superiore al $20 \%$ sui prezzi di listino per i farmaci che non hanno l'obbligo di prescrizione.

L'importanza di tale norma è dovuta al fatto che, mentre il prezzo dei farmaci pagati dal servizio sanitario viene contrattato tra lo stato e il produttore, per quelli a carico del paziente il prezzo è "libero", e può essere rivisto dall' azienda in ogni momento.

Per indurre un risparmio sui farmaci di fascia $\mathrm{C}$ che invece richiedono la ricetta, il decreto ribadisce l'obbligo da parte del farmacista di far presente l'eventuale esistenza di un generico equivalente al farmaco prescritto dal medico e consigliarne l'acquisto (nel caso in cui il medico non abbia scritto sulla ricetta "non sostituibile”). L'Agenzia Italiana del Farmaco (AIFA), in osservanza a tale Decreto Legge, ha stilato e pubblicato un elenco di tutti i farmaci a carico del cittadino con obbligo di prescrizione medica, con i corrispondenti medicinali equivalenti, se esistenti, a minor prezzo. Tale elenco, detto "lista di trasparenza", comprende 3.953 specialità medicinali raggruppate in base al principio attivo ed elencate in ordine crescente di prezzo per unità posologica. $\mathrm{Al}$ fine di garantire ai cittadini un accesso semplice e rapido alle informazioni contenute in tale lista è stato concordato con medici e farmacisti di promuovere la massima diffusione della stessa (apposizione di locandine con il numero verde a cui chiedere informazioni, pubblica-

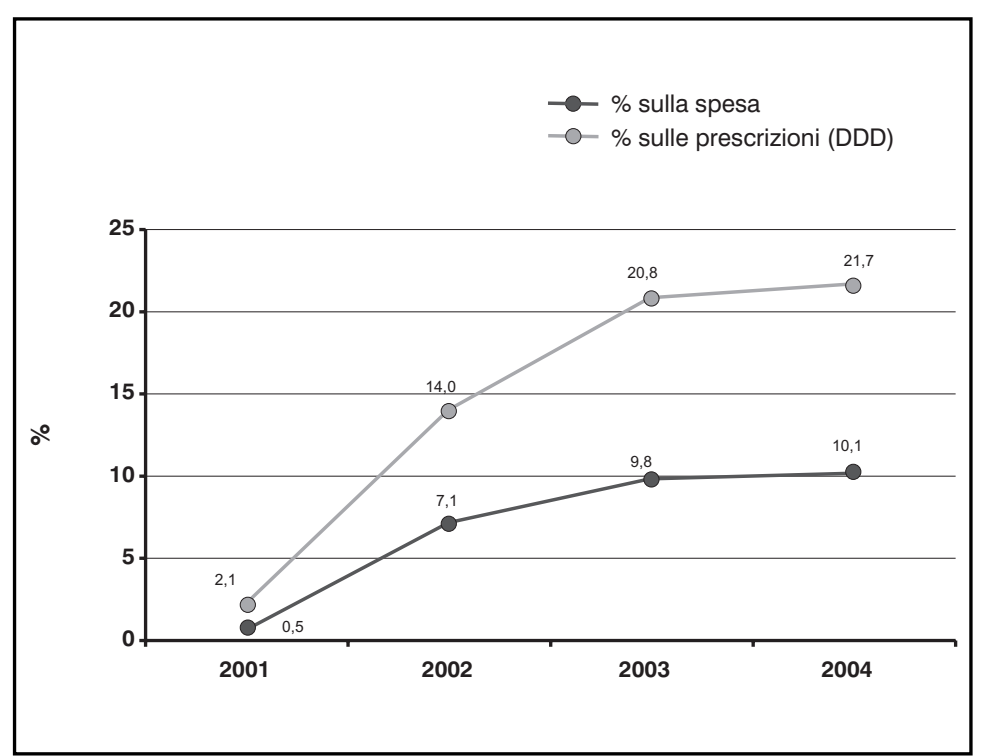

Figura 1

Evoluzione del mercato dei generici (modificata da [36]) zione della lista di trasparenza sul sito dell'AIFA, aggiornamenti in tempo reale delle eventuali variazioni).

Nonostante il mercato dei generici in Italia sia attivo da quasi cinque anni, ha acquisito ad oggi solo limitate quote di mercato e circa il circa il 30\% degli italiani ancora non conosce tali medicinali.

In termini di spesa, il mercato del medicinale equivalente è passato dallo $0,5 \%-1 \%$ nel 2001 al $10,1 \%$ nel 2004 e le prescrizioni sono passate dal $2,1 \%$ al $21,7 \%$ Il ricorso a farmaci a brevetto scaduto risulta sostanzialmente stabile, con una percentuale di DDD (dosi definite die) consumate pari a $20,8 \%$ nel 2003 e a $21,7 \%$ del 2004 (Figura 1).

Il maggior consumo di farmaci equivalenti però non è necessariamente attribuibile ad un incremento delle prescrizioni, può anche essere dovuto a un aumento dei principi attivi con brevetto scaduto.

Nonostante le difficoltà di diffusione dei generici evidenziate in precedenza, l'introduzione di questi farmaci ha rivelato nel tempo una dinamica di riduzione dei prezzi su base concorrenziale discretamente efficace, comportando per alcuni prodotti un abbattimento del prezzo iniziale di oltre il $50 \%$.

In Tabella III abbiamo valorizzato il costo trattamento/die, per un paziente affetto da disturbi d'ansia trattato con alcune delle benzodiazepine disponibili come generici nel nostro Paese. Il calcolo è stato svolto sulla base delle DDD [37], in riferimento ai prezzi attuali del farmaco originatore e del generico più economico. Le copie, o generici branded, non sono state inserite in Tabella (per l'elenco completo vedere la lista di trasparenza dei medicinali in fascia C [38]). Sono stati anche riportati i costi giornalieri della terapia benzodiazepinica calcolati in base ai prezzi del farmaco originatore vigenti prima dell'introduzione dei generici, al fine di valutare se l'introduzione di questi ultimi abbia innescato o meno il meccanismo sperato di riduzione dei prezzi.

Esaminando le differenze percentuali fra il costo giornaliero del farmaco generico e quello del farmaco originatore (Tabella III, colonna 6), possiamo notare come la possibilità di utilizzare il farmaco generico abbia permesso un abbattimento notevole del costo di terapia, da un minimo del $20 \%$ fino ad oltre il $46 \%$ nel caso del lorazepam. Inoltre, il divario fra il costo del farmaco originatore e quello del generico non è diminuito nel corso del tempo, bensì nella maggior parte dei casi è aumentato (Tabella III, colonna 8). In altri termini, lo sviluppo del mercato dei generici non ha sortito appieno l'effetto sperato cioè lo sviluppo di meccanismi concorrenziali in grado di indurre l'abbattimento dei prezzi anche per il farmaco-brand. Questo è spiegabile probabilmente con il fatto che le aziende produttrici di tali farmaci, forti del 
marchio conosciuto, non hanno ritenuto opportuno entrare in concorrenza con le aziende produttrici di farmaci equivalenti.

Anche se la spesa per farmaci rappresenta solo una parte del costo complessivo delle patologie legate all'ansia, occorre tenere in considerazione che, dal momento che le benzodiazepine sono a carico del privato e che spesso vengono assunte ciclicamente e per periodi lunghi, una riduzione della spesa farmacologica può rappresentare per il paziente un risparmio significativo.

\section{CONCLUSIONI}

Fin dalla loro introduzione negli anni '60, le benzodiazepine sono state tra i farmaci più prescritti in assoluto, grazie alla loro elevata efficacia ansiolitica e al profilo di sicurezza che non aveva paragoni tra i farmaci psicoattivi.

Nonostante le principali linee guida, attualmente raccomandino l'uso di inibitori selettivi del reuptake di serotonina o di altri agenti antidepressivi nel trattamento a lungo termine del disturbo d'ansia generalizzato e da attacchi di panico, le benzodiazepine sono ancora di grande utilità nel trattamento delle fasi iniziali di questi disturbi, grazie al rapido e potente sollievo dei sintomi indotto da questi farmaci.

Le benzodiazepine inoltre rimangono il trattamento di prima scelta per una vasta gamma di condizioni cliniche caratterizzate dalla presenza di ansia (ma che non soddisfano i criteri per la diagnosi di un disturbo psichiatrico ben definito), oltre che come ipnotici e sedativi, rapidamente attivi e ben tollerati.

Nell'ambito delle patologie a base ansiosa, ultimamente è stata rivolta particolare attenzione alle presentazioni atipiche e sottosoglia: tra queste si distinguono principalmente i quadri somatomorfi, in cui prevalgono le somatizzazioni, quelli "situazionali", in cui la comparsa dell' ansia è collegata a specifici fattori ambientali o contestuali e i quadri ansiosi secondari a patologie organiche.

Tutte queste forme cliniche non necessitano di una terapia continuativa e a lungo termine, ma traggono beneficio dalla rapida azione ansiolitica delle benzodiazepine.

La collocazione delle benzodiazepine in fascia $\mathrm{C}$, fa sì che il costo della terapia sia interamente a carico del paziente; tale spesa può essere notevolmente ridotta mediante

\begin{tabular}{|c|c|c|c|c|c|c|c|}
\hline Farmaco & Confezione & DDD & $\begin{array}{l}\text { Costo } \\
\text { giornaliero } \\
\text { generico } \\
\text { (euro) }\end{array}$ & $\begin{array}{l}\text { Costo giornaliero } \\
\text { attuale farmaco } \\
\text { originatore } \\
\text { (euro) }\end{array}$ & $\begin{array}{c}\text { Differenza } \\
\text { costo generico } \\
\text { vs } \\
\text { originatore }\end{array}$ & $\begin{array}{l}\text { Costo } \\
\text { giornaliero } \\
\text { pre- } \\
\text { generico } \\
\text { (euro) } \\
\end{array}$ & $\begin{array}{c}\text { Variazione } \\
\text { costo } \\
\text { originatore } \\
\text { dopo l'uscita } \\
\text { del generico } \\
\end{array}$ \\
\hline Alprazolam & $\begin{array}{l}20 \mathrm{cpr} \\
\text { da } 1 \mathrm{mg}\end{array}$ & $1 \mathrm{mg}$ & $\begin{array}{c}\text { 0,35 } \\
\text { (Alprazolam-Teva, Merck } \\
\text { Generics, Abc, Eg, } \\
\text { Hexal, Ratiopharm, } \\
\text { Sandoz) }\end{array}$ & $\begin{array}{c}0,58 \\
\text { (Xanax-Pharmacia) }\end{array}$ & $-39,6 \%$ & 0,53 & $+9,4 \%$ \\
\hline Bromazepam & $\begin{array}{l}\text { gtt } 20 \mathrm{ml} \\
\text { da } 2,5 \mathrm{mg} / \mathrm{ml}\end{array}$ & 10 mg & $\begin{array}{c}\text { 1,00 } \\
\text { (Bromazepam-Teva, } \\
\text { Sandoz, Ratiopharm, } \\
\text { Hexal, Abc, Eg) }\end{array}$ & $\begin{array}{c}1,44 \\
\text { (Lexotan-Roche) }\end{array}$ & $-30,6 \%$ & 1,14 & $+26,3 \%$ \\
\hline Diazepam & $\begin{array}{l}\text { gtt } 20 \\
\mathrm{ml} 5 \mathrm{mg} / \mathrm{ml}\end{array}$ & $10 \mathrm{mg}$ & $\begin{array}{c}0,45 \\
\text { (Diazepam-Merck } \\
\text { Generics, Ratiopharm, } \\
\text { Sandoz) }\end{array}$ & $\begin{array}{c}0,74 \\
\text { (Valium-Roche) }\end{array}$ & $-39,2 \%$ & 0,74 & $=$ \\
\hline Lorazepam & $\begin{array}{l}20 \mathrm{cpr} \\
\text { da } 2,5 \mathrm{mg}\end{array}$ & $2,5 \mathrm{mg}$ & $\begin{array}{c}\text { 0,22 } \\
\text { (Lorazepam-Dorom, Doc, } \\
\text { Teva, Hexal, Ratiopharm, } \\
\text { Merck Generics, Eg, } \\
\text { Sandoz, Abc) }\end{array}$ & $\begin{array}{c}0,41 \\
\text { (Tavor-Wyeth } \\
\text { Lederle) }\end{array}$ & $-46,3 \%$ & 0,35 & $+17,1 \%$ \\
\hline Lormetazepam & $\begin{array}{l}\text { gtt } 20 \mathrm{ml} \\
\text { da } 2,5 \mathrm{mg} / \mathrm{ml} \\
\text { (unica } \\
\text { formulazione } \\
\text { disponibile } \\
\text { come generico) }\end{array}$ & $1 \mathrm{mg}$ & $\begin{array}{c}0,14 \\
\text { (Lormetazepam-Teva, } \\
\text { Ratiopharm, Pliva, Merck } \\
\text { Generics, Hexal, Eg, } \\
\text { Doc, Abc, Allen, Sandoz) }\end{array}$ & $\begin{array}{l}0,18 \\
\text { (Minias-Schering) }\end{array}$ & $-22,2 \%$ & 0,18 & $=$ \\
\hline Triazolam & $\begin{array}{l}20 \mathrm{cpr} \\
0,25 \mathrm{mg}\end{array}$ & $0,25 \mathrm{mg}$ & $\begin{array}{c}\text { 0,42 } \\
\text { (Triazolam-Teva, } \\
\text { Ratiopharm, Pliva, Merck } \\
\text { Generics, Eg, Abc, } \\
\text { Sandoz) }\end{array}$ & $\begin{array}{c}0,52 \\
\text { (Halcion - } \\
\text { Pharmacia Italia) }\end{array}$ & $-19,2 \%$ & 0,52 & $=$ \\
\hline
\end{tabular}

\section{Tabella III}

Confronto tra costo giornaliero della terapia benzodiazepinica in relazione all'uso del farmaco originatore e del farmaco generico, prima e dopo l'introduzione di questi ultimi sul mercato (in caso di più formulazioni disponibili, per omogeneità e praticità, sono state scelte per il calcolo le formulazioni orali, compresse o gocce, con maggior coerenza fra unità posologica e DDD)[39] 
l'utilizzo di farmaci generici, disponibili nel nostro Paese per le principali benzodiazepine utilizzate.

L'introduzione dei farmaci generici ha quindi dato la possibilità al paziente, su consiglio del medico, di scegliere di risparmiare affidandosi all'uso di un farmaco equivalente. L'entità di tale risparmio attualmente varia dal $22 \%$ al $46 \%$ a seconda della molecola ed è quindi notevole, anche in conseguenza al fatto che le aziende di farmaci originatori, forti del marchio e dei pregiudizi del paziente, non solo non hanno ridotto, ma hanno addirittura aumentato $\mathrm{i}$ prezzi di questa classe di farmaci.

Infatti, nonostante lo sforzo informativo svolto in questi anni dal Ministero della Salute e dall' AIFA, il farmaco generico è ancora troppo spesso associato a un'idea di inferiorità in termini di qualità, mentre dovrebbe essere con- siderato come un mezzo equivalente dal punto di vista terapeutico, in grado di generare risorse e di dare un input a meccanismi concorrenziali e, conseguentemente, allo sviluppo di tecniche innovative [36].

L'abbassamento del costo farmacologico può essere considerato di secondaria importanza se paragonato al miglioramento, in termini di qualità di vita e di rendimento dal punto di vista lavorativo, che un paziente può ottenere mediante un opportuno controllo delle patologie a base ansiosa.

Oltre alla riduzione dei costi indiretti, un adeguato trattamento farmacologico permette un consistente risparmio in termini di risorse sanitarie dirette, psichiatriche e non, a fronte di un investimento farmaceutico iniziale che, l'introduzione dei generici sul mercato può contribuire a rendere minimo.

\section{BIBLIOGRAFIA}

1. Judd LL et al. Comorbidity as a fundamental feature of generalised anxiety disorders: results from the national comorbidity study (NCS). Acta Psychiatr Scand 1998;98(suppl 393):6-11.

2. Andrews G ET AL. A comparison of two structured diagnostic interviews: CIDI and SCAN. Aust N Z J Psychiatry 1995;29:124-132.

3. Regier DA et al. Prevalence of anxiety disorders and their comorbidity with mood and addictive disorders.Br $\mathrm{J}$ Psychiatry Suppl. 1998;(34):24-8.

4. Weissman MM et al. The cross-national epidemiology of panic disorder. Arch Gen Psychiatry. 1997 Apr;54(4):305-9.

5. Jessker RC et al. Lifetime and 12-month prevalence of DSM-III-R psychiatric disorders in the United States: results from the national comorbidity survey. Arch Gen Psychiatry 1992;51:8-19.

6. Seivewright $\mathrm{N}$ et al. Longitudinal study of the influence of life events and personality status on diagnostic change in three neurotic disorders. Depression Anx 2000;11:105-113.

7. Pigott T. Gendere differences in the epidemiology and treatment of anxiety disorders. J Clin Psychiatry 1999;60(suppl 18):15-18.

8. Jorm AF. Does old age reduce the risk of anxiety and depression? A review of epidemiological studies across the adult life span. Psych Med 2000;30:11-22.

9. Robins LN, Regier DA, eds. Psychiatric Disorders in America: The Epidemiologic Catchment Area Study. New York, NY: The Free Press, 1991.

10. Andrews G, Henderson S, Hall W. Prevalence, comorbidity, disability and service utilisation. Overview of the Australian National Mental Health Survey. Br J Psychiatry 2001;178:145-153.

11. Greenberg PE et al. The economic burden of anxiety disorders in the 1990s. J Clin Psychiatry. 1999 Jul;60(7):427-35.

12. Sito web dell'Associazione per la Ricerca sulla Depressione - Pubblicazioni on-line (www.depressione-ansia.it). 
13. Practice guideline for psychiatric evaluation of adults. American Psychiatric Association - Medical Specialty Society. 1995 (reviewed 2001).

14. Tallman JF et al. Receptors for the age of anxiety: pharmacology of the benzodiazepines. Science 207:274-281, 1980.

15. Stahl SM. Psicofarmacologia essenziale - Basi neuroscientifiche e applicazioni pratiche. $2^{\circ}$ Ed., Cap 8: 297-333, 2002, Centro Scientifico Editore, Torino.

16. Pancheri P, Torta R, Bigio G, et al, Ravizza L (a cura di). Farmaci attivi nella terapia dell'ansia in Cassano GB, Pancheri P, Pavan L, et al. Trattato Italiano di Psichiatria, $2^{\circ}$ Ed., Vol III, Cap. 97: 3227-3278,1999, Masson, Milano

17. Bellantuono C, Ruggeri M, Piccinelli M. Gli psicofarmaci nella terapia delle sindromi ansiose. In Bellantuono C, Tansella M ( a cura di ). Gli psicofarmaci nella pratica terapeutica, 1994, Il Pensiero Scientifico Editore, Roma

18. Mendelson WB. Clinical distinction between long acting and short acting benzodiazepines. J. Clin. Psychiatry, 60 (Suppl. 22), 12-17, 1992.

19. Hobbs WR, Rall TW, Verdoorn TA. Ipnotici e sedativi. In Goodman Gilman A, Hardman JG, Limbird LE: Le basi farmacologiche della terapia, $10^{\circ}$ ed. Italiana, Vol.1, Cap. 17, 385-412, 2003. The McGraw-Hill Companies Publishing Group Italia, Milano.

20. Fontane R, Chouinard G, Annable L. Rebound anxiety in anxious patients after abrupt withdrawal of benzodiazepine treatment. Am J Psychiatry, 141: 848-852, 1984

21. Hales RE, Yudofsky SC, Talbott JA. Psichiatria (terza edizione italiana del textbook of psychiatry). Cap $27: 1025$ $1111,2003$.

22. DuPont RL et al. Economic costs of anxiety disorders. Anxiety. 1996;2(4):167-72.

23. Mendlowicz MV, Stein MB. Quality of life in individuals with anxiety disorders. Am J Psychiatry 2000;157:669-682.

24. Sherbourne CD, Wells KB, Judd LL. Functioning and well-being of patients with panic disorder. Am J Psychiatry. 1996 Feb;153(2):213-8.

25. Schonfeld WH et al. The functioning and well-being of patients with unrecognized anxiety disorders and major depressive disorder. J Affect Disord. 1997 Apr;43(2):105-19

26. Candilis PJ et al. Quality of life in patients with panic disorder. J Nerv Ment Dis. 1999 Jul;187(7):429-34.

27. Hollifield $\mathrm{M}$ et al. Panic disorder and quality of life: variables predictive of functional impairment. Am J Psychiatry. 1997 Jun;154(6):766-72.

28. Ettigi $\mathrm{P}$ et al. The quality of life and employment in panic disorder. J Nerv Ment Dis. 1997 Jun;185(6):368-72.

29. Leon AC et al. Assessing impairment in patients with panic disorder: the Sheehan Disability Scale. Soc Psychiatry Psychiatr Epidemiol. 1992 Mar;27(2):78-82.

30. Endicott J et al. Quality of Life Enjoyment and Satisfaction Questionnaire: a new measure. Psychopharmacol Bull. 1993;29(2):321-6.

31. http://www.depressione-ansia.it/pdf/approfondimenti/male_curabile.pdf

32. Zambori J et al. Cost-outcome of anxiety treatment intervention in primary care in Hungary. J Ment Health Policy Econ. 2002 Sep;5(3):115-20.

33. Janiri L, Gobbi G. Abuso e dipendenza da benzodiazepine. Istituto di Psichiatria e Psicologia, Università Cattolica del Sacro Cuore - Roma http://www.nemoss.org/uploads/00000070/abuso\%20e\%20dipendenza\%20benzodiazepine.pdf

34. Midha KK, Hubbard JW. Aims and consequences of bioequivalence studies. In: Midha KK, Blume HH. (eds.): BioInternational 2. Bioavailability, Bioequivalence and Pharmacokinetics. Medpharm Scientific Publications, Stuttgart, pp.29-34(1995).

35. Eandi M, Della Pepa C. I farmaci "generici” in Italia: opportunità di ricerca e sviluppo di prodotti di qualità a prezzi competitivi. Farmeconomia e percorsi terapeutici 2003; 4 (2): 65-76.

36. Bollettino d'informazione sui farmaci, 116 http://www.ministerosalute.it/imgs/C_17_bif_bollettino_29_file_itemName_6_filePdf.pdf

37. WHO Collaborating Centre for Drug Statistics Methodology. http://www.whocc.no/atcddd/

38. Liste di trasparenza per i farmaci di fascia C soggetti a prescrizione medica. http://www.ministerosalute.it/medicinali/ bancadati/SceltaFarmaco.jsp (Ultima consultazione il 14.12.2005)

39. SW Compendio Farmaceutico Telematico. Farmadati Italia S.r.l. (Ultima consultazione il 16/12/2005) 